\title{
Elusive Accretion Discs in Low Luminosity AGN
}

\author{
J.A. Fernández-Ontiveros ${ }^{1,2}$, M.A. Prieto Escudero ${ }^{1,2}$, S. Markoff ${ }^{3}$, \\ L. Reb ${ }^{4}$, D. Espada ${ }^{5,6}$ and O. González-Martín ${ }^{7}$ \\ ${ }^{1}$ Instituto de Astrofísica de Canarias (IAC), E-38205 La Laguna, Tenerife, Spain \\ ${ }^{2}$ Universidad de La Laguna, Dept. Astrofísica, E-38206 La Laguna, Tenerife, Spain \\ ${ }^{3}$ Anton Pannekoek Institute of Astronomy, University of Amsterdam, Science Park 904, 1098 \\ XH Amsterdam, The Netherlands \\ ${ }^{4}$ Ludwig-Maximilians-Universität München, Geschwister-Scholl-Platz 1, 80539 Munich, \\ Germany \\ ${ }^{5}$ Joint ALMA Observatory, Alonso de Cordova 3107, Vitacura, Santiago 763-0355, Chile \\ ${ }^{6}$ Instituto de Radioastronomía y Astrofísica (UNAM), 3-72 (Xangari), 8701, Morelia, México
}

\begin{abstract}
Low luminosity AGN (LLAGN) represent the vast majority of the AGN population in the near universe. However, they show intrinsic differences when compared to the Unified Model scenario, i.e. a lack of both the big blue bump and the IR bump in their spectral energy distribution (SED), which are the signatures of an accretion disk and a torus, respectively. In this work we present SED for a sample of six LLAGN in the nearby Universe obtained from subarcsec resolution observations. The nature of the IR continuum emission is discussed in the context of non-thermal radiation powered by compact jets.
\end{abstract}

\section{Introduction}

The vast majority of active galaxies in the Local Universe are Low Luminosity Active Galactic Nuclei (LLAGN), characterised by low accretion rates and modest luminosities (Ho 2008). However, LLAGN are not just scale-down versions of their bright counterparts, showing significant differences with Seyfert galaxies and quasars: $i$ ) absence of the big blue bump (Ho 1996), signature of the accretion disk in the Spectral Energy Distribution (SED); ii) LLAGN tend to be "radio-loud", showing compact cores and parsec-scale radio jets (Nagar et al. 2005); and iii) they are frequently associated with low-ionisation nuclear emission-line regions (LINERs, Heckman 1980).

Structural changes predicted at low luminosities might explain these differences: the broad-line region and the "torus", keystones of the Unified Model (Antonucci 1993), are expected to vanish at $L_{b o l} \lesssim 10^{42} \mathrm{erg} / \mathrm{s}$ (Laor 2003; Hönig \& Beckert 2007). The absence of the blue bump emission led to the wide use of the advection-dominated accretion solution (ADAF) which has been applied successfully from X-ray binaries to Sgr A* (Narayan et al. 1995), to explain the spectral shape of LLAGN and their low luminosity (Fabian \& Rees 1995; Di Matteo et al. 2003; Yuan et al. 2009; Eracleous et al. 2010). However, ADAF models have been mainly applied to SEDs with scarce wavelength coverage and/or based on flux measurements using large apertures. This is especially critical in the optical and IR ranges, where the host galaxy usually outshines these faint nuclei.

\section{High-angular resolution data}

This work is based on a multiwavelength, high-angular resolution dataset for the inner few kiloparsecs of a sample of six LLAGN. The use of Adaptive Optics (AO) in the nearIR (NACO) and diffraction-limited imaging in the mid-IR (VISIR) at the Very Large 
Telescope (VLT) allowed us to sample the 1-20 $\mu \mathrm{m}$ range with nuclear flux measurements using subarcsec apertures. The dataset was completed with optical/UV imaging with the Hubble Space Telescope (HST) scientific archive. Nuclear fluxes were measured using aperture photometry of the unresolved component in the centre $\left(\lesssim 00^{\prime \prime} 1\right)$, subtracting the local background around $\left(00^{\prime \prime} 2-0 . .3\right)$ in order to remove the contribution of stars and dust in the host galaxy. In the mid-IR, PSF photometry was taken from Reunanen et al. (2010); Asmus et al. (2014). Measurements in the radio and X-ray ranges have been collected after an extensive and careful search in the literature. These correspond mainly to Very Large Array (VLA) and Very Long Baseline Interferometry (VLBI) in radio and Chandra, XMM-Newton and Integral at X-rays. Despite of their lower spatial resolution, the flux at high energies is dominated by the AGN and not by the host galaxy, and thus can be consistently compared with subarcsec measurements at other wavelength ranges. The characteristics of this dataset allow us to build, for each LLAGN in the sample, a consistent SED of the same physical region, very well sampled over a wide range in wavelength. Additionally, a low-spatial resolution SED based on apertures larger than few arcsec was also built using the NED database $\dagger$ and measurements published in the literature. The low-spatial resolution data is complementary to the subarcsec SED, as they permit to identify those spectral ranges in which the host galaxy shows a strong contribution, that might contaminate the nuclear spectrum.

This project is part of The central parsecs of the nearest galaxies $\ddagger$ (Reunanen et al. 2010; Prieto et al. 2010). The objects included in the present work correspond to the nearest LLAGN accessible from the Southern Hemisphere. Three of the objects are the canonical reference for definition of the LLAGN class (NGC 1052 in Heckman 1980; NGC 1097 in Keel 1983; M87 in Fabian \& Rees 1995).

\subsection{The nature of the IR emission}

The multiwavelength high-angular resolution SED allow us to: $i$ ) probe the genuine SED of the LLAGN; and ii) identify the physical mechanisms that dominate the energy output in different wavelength ranges. Fig. 1 shows the subarcsec SEDs for the sample of LLAGN (black dots), sorted by increasing distance. Low-spatial resolution data (grey spikes) trace the contribution of the host galaxy, which dominates the overall emission in most of the cases. For comparison, the radio-loud quasar template from Elvis et al. (1994) is also shown (grey-solid line). The self-absorbed synchrotron emission is represented as a broken power law (see below Marscher \& Gear 1985), which is fitted to the radio-to-UV range for each subarcsec aperture SED (dashed line).

\section{2. $L L A G N$ at parsec scales}

The SEDs of LLAGN in our sample show: $i$ ) a flat radio continuum, more similar to radioloud quasars rather than Seyfert nuclei; ii) mid-IR and X-ray ranges drive the bolometric luminosity of these nuclei; and iii) subarcsec SEDs show a flat-to-inverted shape from radio to IR/optical wavelengths, which is characteristic of non-thermal emission in a jet. Note the steep slope in the IR/optical continuum $\left(1<\alpha<3 ; S_{\nu} \propto \nu^{-\alpha}\right)$, far from the classical synchrotron cooling slope of $\alpha \approx 0.7$. The Sombrero galaxy (NGC 4594) shows a soft thermal bump at $\sim 1 \mu$ m that might be caused by a receding accretion disc.

At high-angular resolution, LLAGN show significantly different SEDs when compared with quasars and Seyfert galaxies. The torus, if present, is not evidenced by the continuum emission, which seems to follow a non-thermal distribution (Fig. 1). Radio-loud quasars are more similar to LLAGN in the radio and X-ray ranges, but the absence of a big blue

$\dagger$ http://ned.ipac.caltech.edu/

$\ddagger$ http://www.iac.es/project/parsec/ 

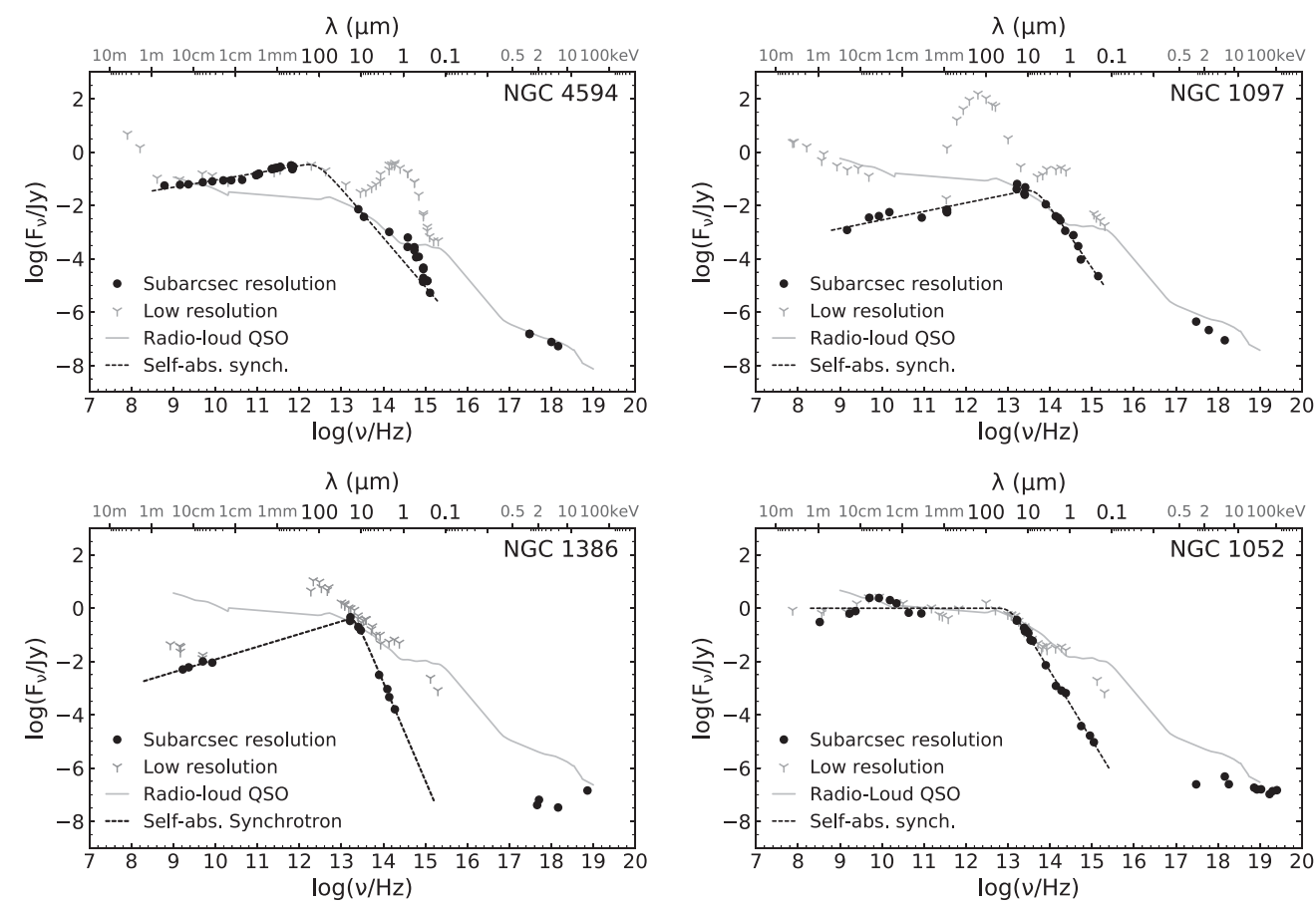

Figure 1. Subarcsec SEDs (black dots) and low-spatial resolution data (grey spikes) for four of the LLAGN in our sample. The grey line corresponds to the radio-loud quasar template from Elvis et al. (1994). A broken power law, i.e. representative of self-absorbed synchrotron emission from a jet, has been fitted to the radio, IR and optical/UV ranges (dashed line).

bump in the latter suggest important structural differences between both classes. The LLAGN in our sample are in agreement with the optical to X-ray colours and the IR to X-ray correlation from Maoz (2007); Asmus et al. (2011). However, this behaviour is not produced by thermal features in the SED. In contrast, both spectral slopes are produced by a steep power-law continuum from the mid-IR to optical/UV wavelengths. The nonthermal behaviour can be well described, at first order, by a self-absorbed synchrotron model (dashed line in Fig. 1 Marscher \& Gear 1985), suggesting a jet as the component that drives the overall continuum emission in these objects. In Section 3 we further explore this possibility using a more detailed model of a jet. Nonetheless, one should keep in mind that spectral ratios are not able to discriminate bright AGN with strong thermal contributions in the mid-IR (torus) and the optical/UV (accretion disk) from LLAGN dominated by a steep non-thermal continuum as those included in our work.

\section{Jet model}

Data for M87 are carefully selected from quiescent periods in which the core was in a quiet state of activity (see Fig. 2, left panel), plus an active period between 2005-2006 during which the nucleus and the jet knot HST-1 were in a loud state, see Prieto et al. (2016) for a detailed discussion on the M87 data. The right panel in Fig. 2 shows a fit of the jet+disk model developed by S. Markoff (Markoff et al. 2005, 2008) to the SED of M87. The jet emission can explain the overall shape of the continuum emission for this LLAGN. 

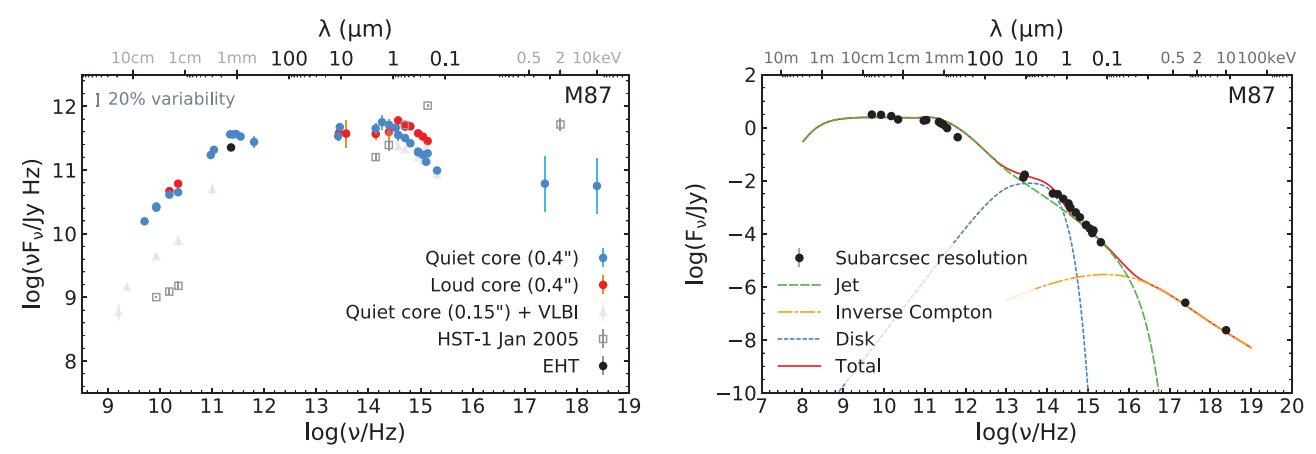

Figure 2. Left: Subarcsec resolution SED for the quiet and loud states of M87. Right: jet+disk model from Markoff et al. (2005, 2008), applied to the subarcsec SED of M87. Individual components of the model are also plotted. A colour version of this figure is available in the electronic version of this journal.

\section{Summary}

The LLAGN in our sample show remarkably homogeneous SEDs at subarcsec resolution. The torus and the big blue bump, if present, are not detected. Most of the nuclei show a featureless shape well described by a flat-to-inverted continuum from radio to the optical range, favouring a compact jet as the dominant source of the continuum emission. However, the IR/optical continuum does not follow the classical synchrotron cooling slope, but shows instead a much steeper spectrum $(1<\alpha<3)$. Very high radiative losses or a thermal lepton distribution at the base of the jet can explain this behaviour.

\section{References}

Antonucci, R. 1993, ARA\&A, 31, 473

Asmus, D., Gandhi, P., Smette, A., Hönig, S. F., \& Duschl, W. J. 2011, A\&A, 536, A36

Asmus, D., Hönig, S. F., Gandhi, P., Smette, A., \& Duschl, W. J. 2014, MNRAS, 439, 1648

Di Matteo, T., Allen, S. W., Fabian, A. C., Wilson, A. S., \& Young, A. J. 2003, ApJ, 582, 133

Elvis, M., Wilkes, B. J., McDowell, J. C., et al. 1994, ApJS, 95, 1

Eracleous, M., Hwang, J. A., \& Flohic, H. M. L. G. 2010, ApJS, 187, 135

Fabian, A. C. \& Rees, M. J. 1995, MNRAS, 277, L55

Heckman, T. M. 1980, A\&A, 87, 152

Ho, L. C. 1996, PASP, 108, 637

Ho, L. C. 2008, ARA\&A, 46, 475

Hönig, S. F. \& Beckert, T. 2007, MNRAS, 380, 1172

Keel, W. C. 1983, ApJ, 269, 466

Laor, A. 2003, ApJ, 590, 86

Maoz, D. 2007, MNRAS, 377, 1696

Markoff, S., Nowak, M., Young, A., et al. 2008, ApJ, 681, 905

Markoff, S., Nowak, M. A., \& Wilms, J. 2005, ApJ, 635, 1203

Marscher, A. P. \& Gear, W. K. 1985, ApJ, 298, 114

Nagar, N. M., Falcke, H., \& Wilson, A. S. 2005, A\&A, 435, 521

Narayan, R., Yi, I., \& Mahadevan, R. 1995, Nature, 374, 623

Prieto, M. A., Fernández-Ontiveros, J. A., Markoff, S., Espada, D., \& González-Martín, O. 2016, MNRAS, 457, 3801

Prieto, M. A., Reunanen, J., Tristram, K. R. W., et al. 2010, MNRAS, 402, 724

Reunanen, J., Prieto, M. A., \& Siebenmorgen, R. 2010, MNRAS, 402, 879

Yuan, F., Yu, Z., \& Ho, L. C. 2009, ApJ, 703, 1034 